http://dx.doi.org/10.9789

\title{
Indicadores bibliométricos de colaboração científica na área de enfermagem do trabalho \\ Bibliometric indicators of scientific collaboration in the field of occupational nursing

\author{
Indicadores bibliométricos de colaboración científica en el campo de la enfermería \\ ocupacional
}

\section{RESUMO}

\author{
${ }^{1}$ Viviane Reis Fontes da Silva, ${ }^{2}$ Priscila Bastos Mayworm
}

\begin{abstract}
Objetivo: específicos: determinar a posição da colaboração científica da produção da enfermagem do trabalho do Brasil no cenário da América latina e; mapear a colaboração e a produção científica na área de enfermagem do trabalho na perspectiva da qualidade total. Método: bibliométrico/cientométrico da produção científica na área de enfermagem do trabalho. A obtenção dos dados bibliométricos ocorreu no mês de fevereiro de 2014, sendo a fonte a rede mundial de computadores a partir do portal de periódicos da Coordenação de Aperfeiçoamento de Pessoal de Nível Superior - Capes. Resultados: A obtenção dos dados bibliométricos ocorreu no mês de fevereiro de 2014, sendo a fonte a rede mundial de computadores, a partir do portal de periódicos da Coordenação de Aperfeiçoamento de Pessoal de Nível Superior - Capes, Conselho Nacional de Desenvolvimento Científico e Tecnológico CNPq e as bases de dados SCOPUS e SCIMAGO. Os termos utilizados como descritores para busca online, inicialmente, foram os acima já descritos, obedecendo uma ordem hierárquica a partir do descritor "enfermagem". Conclusão: O Brasil mostra um baixo desempenho quando a busca ocorre com o descritor "enfermagem do trabalho e qualidade total". Com este descritor a enfermagem brasileira deixa o top ten. Isso mostra que a referente qualidade total na perspectiva da enfermagem do trabalho ainda precisa ser melhor e mais abordada/pesquisada nas instituições de ensino e pesquisa, principalmente, nos cursos de pós-graduação - mestrado e doutorado. DESCRITORES: Enfermagem do trabalho; Qualidade total; Cientometria.
\end{abstract}

\section{ABSTRACT}

Objective: specific: to determine the position of scientific collaboration in the production of nursing work in Brazil in the Latin American scenario and; to map collaboration and scientific production in the field of occupational nursing from the perspective of total quality. Method: bibliometric / scientometric of scientific production in the field of occupational nursing. The collection of bibliometric data took place in February 2014, the source being the world wide web from the journals portal of the Coordination for the Improvement of Higher Education Personnel - Capes. Results: Bibliometric data were obtained in February 2014, the source being the world wide web, from the periodical portal of the Coordination for the Improvement of Higher Education Personnel - Capes, CNPq National Council for Scientific and Technological Development and the SCOPUS and SCIMAGO databases. The terms used as descriptors for online search, initially, were the ones already described above, obeying a hierarchical order from the descriptor "nursing". Conclusion: Brazil shows a low performance when the search occurs with the descriptor "nursing work and total quality". With this descriptor, Brazilian nursing leaves the top ten. This shows that the referent total quality from the perspective of occupational nursing still needs to be better and more addressed / researched in teaching and research institutions, especially in graduate courses - master's and doctorate. DESCRIPTORS: Occupational nursing; Total quality; Scientometry.

\section{RESUMEN}

Objetivo: específico: determinar la posición de la colaboración científica en la producción de trabajo de enfermería en Brasil en el escenario latinoamericano y; mapear la colaboración y la producción científica en el campo de la enfermería ocupacional desde la perspectiva de la calidad total. Método:

\footnotetext{
${ }^{1}$ Enfermeira da Secretaria Municipal de Saúde - RJ. Mestre em Enfermagem Universidade Federal do Estado do Rio de Janeiro - UNIRIO.

${ }^{2}$ Enfermeira do Hospital Cardoso Fontes e da Prefeitura de Arraial do Cabo, no Estado do Rio de Janeiro.
}

Rev. Eletr de Cien. Tecnol e Inova, Rio de Janeiro, Vol. 10: 1-24, abril/2020. 
bibliométrico / scientometric de producción científica en el campo de la enfermería ocupacional. La recopilación de datos bibliométricos tuvo lugar en febrero de 2014, siendo la fuente la red mundial del portal de revistas de la Coordinación para la Mejora del Personal de Educación Superior - Capes. Resultados: La recopilación de datos bibliométricos se realizó en febrero de 2014, siendo la fuente la red mundial, del portal periódico de la Coordinación para la Mejora del Personal de Educación Superior - Capes, Consejo Nacional de Desarrollo Científico y Tecnológico CNPq y las bases de datos SCOPUS y SCIMAGO. Los términos utilizados como descriptores para la búsqueda en línea, inicialmente, fueron los ya descritos anteriormente, obedeciendo un orden jerárquico del descriptor "enfermería". Conclusión: Brasil muestra un bajo desempeño cuando la búsqueda ocurre con el descriptor "trabajo de enfermería y calidad total". Con este descriptor, la enfermería brasileña deja el top ten. Esto muestra que la calidad total de referencia desde la perspectiva de la enfermería ocupacional todavía necesita ser mejor y más abordada / investigada en las instituciones de enseñanza e investigación, especialmente en los cursos de posgrado: maestría y doctorado. DESCRIPTORES: Enfermería ocupacional; Calidad total; Scientometry.

\section{INTRODUÇÃO}

A enfermagem é uma profissão jovem quando comparada a outras pertencentes ou não a mesma área de conhecimento, entretanto, seu crescimento tem ocorrido tanto na pragmática assistencial como também, no plano da investigação científica.

A constante atualização nasce, dentre outras coisas, da necessidade de acompanhar os avanços da profissão e crescimento da produção científica em enfermagem e áreas correlatas, sendo que este último precisa, além do caráter quantitativo, aperfeiçoar ainda mais e principalmente, o caráter qualitativo. ${ }^{1}$

A Enfermagem é um campo de conhecimento específico e se configura como profissão social que se consolida, expande e se fortalece como ciência, tecnologia e inovação. Não obstante, seu objeto de investigação é de extrema relevância social: o cuidado de enfermagem na saúde humana; cabe aqui ressaltar que este cuidado transcende àqueles

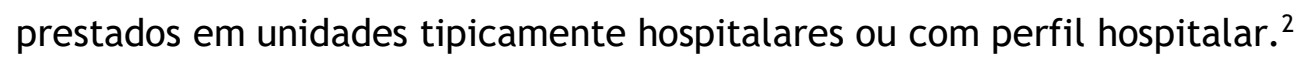

Dados da Organização Mundial de Saúde proporcionaram sua visibilidade internacional ao ponto de, em 2010, essa mesma Organização chamasse atenção para o Ano Internacional da Enfermagem.

No plano da ação, a enfermagem representa, aproximadamente, $60 \%$ dos profissionais da área da saúde, no Sistema Único de Saúde do País, aproximadamente 1,3 milhões de trabalhadores da enfermagem, mostrando-se resolutiva e contributiva para a promoção e manutenção da saúde dos brasileiros.

Rev. Eletr de Cien. Tecnol e Inova, Rio de Janeiro, Vol. 10: 1-24, abril/2020. 
No plano da investigação, a enfermagem também apresenta significativa contribuição para a melhoria dos indicadores da produção científica do Brasil, haja vista que contabilizamos mais de 900 cursos de graduação em enfermagem em nosso país.

No âmbito da Pós-Graduação, a enfermagem brasileira vem se consolidando cada vez mais na formação de recursos humanos em pesquisa com a titulação de mestres e de doutores, respectivamente, desde as décadas de 70 e 80 , fortalecendo, nas suas especificidades, além de articular e integrar conhecimentos com outros setores determinantes da vida, e também daqueles comprometidos diretamente com a saúde, mas, que contribuem para o ser humano em sua complexidade e totalidade, como é o caso da saúde na área do trabalhador. ${ }^{3}$

Portanto, é nesse exato momento que vislumbramos e reconhecemos a importância de os serviços de enfermagem do trabalho voltar sua atenção para a gestão pela qualidade total. Não obstante, os enfermeiros que militam na enfermagem do trabalho buscam cada vez mais a qualificação necessária de maneira que sua contribuição seja efetivamente assentada em bases sólidas de conhecimento e que este conhecimento seja revertido na pragmática assistencial no cotidiano de enfermeiros e enfermeiras que assistem os trabalhadores nas mais diferentes frentes organizacionais.

Os programas de pós-graduação têm feito a sua parte. Atualmente, o cenário nacional dispõe de 41 programas de Pós-Graduação stricto sensu em Enfermagem, todos credenciados pela Coordenação de Aperfeiçoamento de Pessoal de Nível Superior - CAPES, distribuídos em 38 Cursos de mestrado Acadêmico, 21 Cursos de Doutorado e 3 na modalidade de Mestrado profissional.

Esses Programas tornam possível a formação qualificada de recursos humanos de enfermagem nas mais diversas áreas, inclusive, na enfermagem do trabalho e/ou com vistas à saúde do trabalhador tal como objeto de investigação, com a atuação de Grupos de 
Pesquisa produzindo conhecimento em prol da melhoria da saúde e do bem-estar da população.

Pesquisa é toda atividade voltada para a solução de problemas, como atividades de busca, indagação, investigação, inquirição da realidade. É a atividade que nos vai permitir, no âmbito da ciência, elaborar um conhecimento ou conjunto de conhecimentos que nos auxilie na compreensão desta realidade e nos oriente em nossas ações.

O termo Bibliometria foi criado em 1934 por Paul Outlet, sendo antes esta ciência conhecida como bibliografia estatística, termo gravado por Hulme. No entanto, o termo consolidou-se apenas em 1969, após a publicação do artigo de Pritchard, sob o título “Bibliografia estatística ou Bibliometria?”. ${ }^{4-5}$

O estudo bibliométrico consiste na aplicação da estatística à bibliografia. Além disso, pode ter classificações como os estudos macrobiliométricos ou nacionais, que compreendem a análise estatística da produção bibliográfica de uma nação, e os estudos denominados microbiliométricos, no qual a análise se reduz à determinada ciência ou conhecimento científico e humanístico.

Destarte, a estatística bibliográfica, isto é, parte do estudo bibliométrico depende também da bibliografia. Surgindo da necessidade de reagrupar as obras, de organizá-las em categoria, obtendo-se indicadores que correspondem às classificações dos estudos realizados, trabalho este que facilita a pesquisa do leitor e do pesquisador. As três Leis básicas da Bibliometria são: a Lei de Bradford (mede produtividade de periódicos), Lei de Lotka (mensura a produtividades de autores) e Lei de Zipf (observa a frequência de ocorrência de palavras). ${ }^{5-8}$

A lei de Bradford é também conhecida como de lei da dispersão e "permite, mediante a medição da produtividade das revistas, estabelecer o núcleo e as áreas de dispersão sobre um determinado assunto em um mesmo conjunto de revistas, em outras palavras, estima o grau de relevância de periódicos em dada área do conhecimento, visto que os periódicos 
com o maior número de artigos sobre dado assunto formam um núcleo de periódicos, supostamente de maior qualidade ou relevância para aquela área. Tem o objetivo de descobrir a extensão na qual artigos de um assunto científico específico apareciam em periódicos destinados a outros assuntos, estudando a distribuição dos artigos em termos de variáveis de proximidade ou de afastamento..$^{6-8}$

Esta Lei sugere ainda que quando artigos com assuntos novos são escritos e submetidos a periódicos apropriados, se aceitos, atraem cada vez mais artigos sobre determinado assunto, fortalecendo o desenvolvimento de pesquisas em determinada área. O enunciado da lei diz que "se os periódicos forem ordenados em ordem de produtividade decrescente de artigos sobre um determinado assunto, poderão ser distribuídos num núcleo de periódicos mais particularmente devotados a esse assunto e em diversos grupos ou zonas contendo o mesmo número de artigos que o núcleo, sempre que o número de periódicos e das zonas sucessivas for igual a 1:n:n²..."6-7

A Lei de Lotka descreve a produtividade de autores e fundamentada na premissa básica de que "alguns pesquisadores publicam muito e muitos publicam pouco", enuncia que “a relação entre o número de autores e o número de artigos publicados por esses, em qualquer área científica, segue a Lei do Inverso do Quadrado $1 / \mathrm{n}^{2}$." A referida Lei foi formulada em 1926, de um estudo sobre a observação da produtividade de cientistas a partir da contagem de autores presentes no Chemical Abstracts (Revista da Sociedade Americana de Química) entre 1909 e 1916.5-8

Lotka concluiu que, muito embora existisse um grande número de autores produzindo na literatura científica, boa parte da produção é realizada por um pequeno número de autores, e, observou também que um grande número de pequenos produtores se iguala, em produção, ao reduzido número de grandes produtores. A partir daí formulou a lei dos quadrados inversos: $y x=6 / p 2 x a$, onde yx é a frequência de autores publicando número $x$ de 
trabalhos e a é um valor constante para cada campo científico (2 para físicos e 1,89 para químicos, por exemplo)..$^{5-8}$

Na gestão da informação, do conhecimento e planejamento científico e tecnológico, sua aplicabilidade se faz necessária ao verificar a avaliação da produtividade de pesquisadores, a identificação dos centros de pesquisa mais desenvolvidos em determinada área demonstra a consolidação desta área, o que demonstra que quanto mais delimitada estiver uma ciência maior a probabilidade de seus autores produzirem múltiplos artigos, em dado período de tempo.

A terceira das leis bibliométricas clássicas é a Lei de Zipf, formulada em 1949 e que descreve a relação entre palavras num determinado texto suficientemente grande e a ordem de série destas palavras (contagem de palavras em largas amostragens). Zipf, analisando a obra Ulisses, de James Joyce, encontrou uma correlação entre o número de palavras diferentes e a frequência de seu uso e concluiu que existe uma regularidade fundamental na seleção e uso das palavras e que um pequeno número de palavras é usado muito mais frequentemente..$^{4-7}$

Zipf descobriu que a palavra mais utilizada aparecia 2653 vezes, a centésima palavra mais utilizada ocorria 256 vezes e a duocentésima palavra ocorria 133 vezes. Zipf viu então que a posição de uma palavra multiplicada pela sua frequência era igual a uma constante de aproximadamente 26500 . Sua proposta, assim, é de que, se listarmos as palavras que ocorrem num texto em ordem decrescente de frequência, a posição de uma palavra na lista multiplicada por sua frequência é igual a uma constante. A equação para esse relacionamento é: $r \times f=k$, onde $r$ é a posição da palavra, $f$ é a sua frequência e $k$ é a constante. ${ }^{4-7}$

\section{MÉTODO}

A metodologia utilizada foi do tipo quantitativa por ser aquela que mais se adéqua ao objeto de nossa investigação. Trata-se de uma avaliação numérica dos dados coletados 
ao longo da pesquisa. A abordagem quantitativa busca a mensuração de variáveis preestabelecidas para verificar e explicar sua influência sobre outras mediante a análise da frequência de incidências e correlações estatística. A obtenção dos dados bibliométricos ocorreu no mês de fevereiro de 2014 , sendo a fonte a rede mundial de computadores, a partir do portal de periódicos da Coordenação de Aperfeiçoamento de Pessoal de Nível Superior - Capes, Conselho Nacional de Desenvolvimento Científico e Tecnológico CNPq e as bases de dados SCOPUS e SCIMAGO. Os termos utilizados como descritores para busca online, inicialmente, foram os acima já descritos, obedecendo uma ordem hierárquica a partir do descritor "enfermagem". ${ }^{4-6}$

\section{RESULTADOS E DISCUSSÃO}

A Tabela 1 mostra todas as grandes áreas da árvore de conhecimento da CAPES desde 2000, com intervalo bianual até o ano de 2010. Muito embora não esteja ocupando o primeiro lugar, perdendo apenas para a área de ciências humanas, fica evidente o crescimento de instituições e grupos de pesquisa cadastrados no CNPq.

\begin{tabular}{|c|c|c|c|c|c|c|c|c|c|c|c|c|}
\hline \multirow{2}{*}{ Grande área (1) } & \multicolumn{6}{|c|}{ Instituições } & \multicolumn{6}{|c|}{ Grupos (G) } \\
\hline & 2000 & 2002 & 2004 & 2006 & 2008 & 2010 & 2000 & 2002 & 2004 & 2006 & 2008 & 2010 \\
\hline Ciências Agrárias & 107 & 124 & 144 & 167 & 181 & 206 & 1.352 & 1.653 & 1.997 & 2.041 & 2.177 & 2.699 \\
\hline Ciências Biológicas & 139 & 164 & 194 & 213 & 224 & 247 & 1.720 & 2.126 & 2.561 & 2.624 & 2.696 & 3.108 \\
\hline Ciências da Saúde & 124 & 156 & 191 & 225 & 250 & 258 & 1.832 & 2.513 & 3.371 & 3.610 & 3.961 & 4.573 \\
\hline Ciências Exatas e da Terra & 138 & 157 & 182 & 203 & 209 & 225 & 1.812 & 2.051 & 2.454 & 2.460 & 2.515 & 2.934 \\
\hline Ciências Humanas & 138 & 160 & 205 & 246 & 262 & 288 & 1.711 & 2.399 & 3.088 & 3.679 & 4.219 & 5.387 \\
\hline Ciências Sociais Aplicadas & 124 & 156 & 196 & 242 & 260 & 282 & 930 & 1.429 & 2.120 & 2.501 & 2.754 & 3.438 \\
\hline Engenharias & 130 & 160 & 194 & 219 & 227 & 249 & 1.826 & 2.243 & 2.826 & 2.844 & 3.027 & 3.548 \\
\hline Linguística, Letras e Artes & 81 & 104 & 128 & 149 & 163 & 163 & 577 & 744 & 1.053 & 1.265 & 1.448 & 1.836 \\
\hline Todas as grandes áreas & 224 & 268 & 335 & 403 & 422 & 452 & 11.760 & 15.158 & 19.470 & 21.024 & 22.797 & 27.523 \\
\hline
\end{tabular}

Fonte: Censos CNPq

Em seguida, na Tabela 2 destaca-se o quantitativo de recursos humanos e linhas de pesquisa segundo a grande área de conhecimento do CNPq envolvendo os pesquisadores doutores. Mais uma vez, a área de saúde perde apenas para a grande área de ciências 
humanas, talvez, pela maior quantidade de sub-áreas e, portanto, com maior quantidade de cursos de pós-graduação, consequentemente, maior quantidade de grupos de pesquisa. 0 mesmo ocorre quando comparamos com o número total de pesquisadores doutores com estudantes, conforme Tabela 3.

\begin{tabular}{|c|c|c|c|c|c|c|c|c|c|c|c|c|}
\hline \multirow{2}{*}{ Grande área (1) } & \multicolumn{6}{|c|}{ Pesquisadores (P) } & \multicolumn{6}{|c|}{ Doutores (D) } \\
\hline & 2000 & 2002 & 2004 & 2006 & 2008 & 2010 & 2000 & 2002 & 2004 & 2006 & 2008 & 2010 \\
\hline Ciências Agrárias & 6.880 & 7.611 & 9.814 & 10.840 & 12.242 & 15.269 & 4.015 & 5.146 & 6.968 & 8.128 & 9.378 & 11.718 \\
\hline Ciências Biológicas & 6.948 & 8.191 & 10.600 & 11.896 & 13.368 & 16.033 & 4.656 & 5.934 & 8.073 & 9.416 & 10.769 & 13.085 \\
\hline Ciências da Saúde & 8.534 & 10.408 & 15.408 & 18.382 & 21.207 & 25.445 & 4.489 & 5.958 & 8.956 & 11.237 & 13.164 & 15.868 \\
\hline Ciências Exatas e da Terra & 7.257 & 7.936 & 10.181 & 10.871 & 11.835 & 14.621 & 5.386 & 6.230 & 8.226 & 8.988 & 9.809 & 11.885 \\
\hline Ciências Humanas & 8.452 & 10.811 & 15.031 & 18.838 & 23.087 & 30.378 & 4.012 & 5.504 & 8.187 & 10.653 & 13.107 & 17.009 \\
\hline Ciências Sociais Aplicadas & 4.408 & 5.843 & 9.444 & 11.987 & 14.429 & 18.579 & 1.948 & 2.854 & 4.876 & 6.355 & 7.600 & 9.720 \\
\hline Engenharias & 8.143 & 9.668 & 13.006 & 13.905 & 15.203 & 18.453 & 4.966 & 6.117 & 8.430 & 9.505 & 10.729 & 12.912 \\
\hline Linguística, Letras e Artes & 2.242 & 2.874 & 4.243 & 5.465 & 6.644 & 8.860 & 1.195 & 1.620 & 2.592 & 3.423 & 4.227 & 5.492 \\
\hline Todas as grandes áreas & 48.781 & 56.891 & 77.649 & 90.320 & 104.018 & 128.892 & 27.662 & 34.349 & 47.973 & 57.586 & 66.785 & 81.726 \\
\hline
\end{tabular}

Fonte: Censos CNPq

\begin{tabular}{|c|c|c|c|c|c|c|c|c|c|c|c|c|}
\hline \multirow{2}{*}{ Grande área (1) } & \multicolumn{6}{|c|}{ Doutores (D) } & \multicolumn{6}{|c|}{ Estudantes (E) } \\
\hline & 2000 & 2002 & 2004 & 2006 & 2008 & 2010 & 2000 & 2002 & 2004 & 2006 & 2008 & 2010 \\
\hline Ciências Agrárias & 4.015 & 5.146 & 6.968 & 8.128 & 9.378 & 11.718 & 6.219 & 6.890 & 11.018 & 13.548 & 19.022 & 24.146 \\
\hline Ciências Biológicas & 4.656 & 5.934 & 8.073 & 9.416 & 10.769 & 13.085 & 11.056 & 11.464 & 17.494 & 20.335 & 25.139 & 29.004 \\
\hline Ciências da Saúde & 4.489 & 5.958 & 8.956 & 11.237 & 13.164 & 15.868 & 8.196 & 8.944 & 15.879 & 21.739 & 31.174 & 38.521 \\
\hline Ciências Exatas e da Terra & 5.386 & 6.230 & 8.226 & 8.988 & 9.809 & 11.885 & 8.646 & 8.303 & 12.563 & 13.944 & 17.489 & 20.591 \\
\hline Ciências Humanas & 4.012 & 5.504 & 8.187 & 10.653 & 13.107 & 17.009 & 8.491 & 9.881 & 17.667 & 24.510 & 35.088 & 43.809 \\
\hline Ciências Sociais Aplicadas & 1.948 & 2.854 & 4.876 & 6.355 & 7.600 & 9.720 & 3.794 & 4.088 & 8.259 & 11.818 & 16.475 & 21.978 \\
\hline Engenharias & 4.966 & 6.117 & 8.430 & 9.505 & 10.729 & 12.912 & 11.649 & 10.827 & 17.332 & 18.947 & 23.050 & 28.264 \\
\hline Linguística, Letras e Artes & 1.195 & 1.620 & 2.592 & 3.423 & 4.227 & 5.492 & 2.202 & 2.770 & 5.094 & 7.126 & 10.265 & 13.088 \\
\hline Todas as grandes áreas & 27.662 & 34.349 & 47.973 & 57.586 & 66.785 & 81.726 & 59.357 & 61.872 & 102.913 & 128.969 & 160.931 & 213.433 \\
\hline
\end{tabular}

Fonte: Censos CNPq

Notas: Grande área predominante do grupo. Não existe dupla contagem no número de pesquisadores, doutores e estudantes tanto em cada grande área como em seus totais, que

Rev. Eletr de Cien. Tecnol e Inova, Rio de Janeiro, Vol. 10: 1-24, abril/2020. 
não foram obtidos por soma; as relações foram calculadas considerando dupla contagem nas diversas dimensões.

Uma parcela da tendência de crescimento observada (grupos, pesquisadores, estudantes, linhas) pode estar relacionada ao aumento do de instituições incluídas nos censos e da taxa de cobertura no âmbito das instituições. Quando analisamos o número de autores e da produção científica dos pesquisadores doutores, segundo a grande área, encontramos no período de 1998 a 2001 uma produção da área da saúde superior a das ciências humanas, enquanto a Saúde, num total de autores publicou 13.189 trabalhos completos em anais de eventos, as Humanas publicou 13.632, sendo ambas superadas pelas engenharias que publicou 60.319 trabalhos.

No entanto, quando mudamos o escopo da publicação e passamos então para a publicação de livros e capítulos de livros, a saúde é que apresenta a maior produção entre as três, o mesmo ocorrendo com a publicação de artigos completos publicados em periódicos especializados no âmbito nacional e internacional. No entanto, quando comparada com as ciências Exatas e da Terra, a Saúde não apresenta o mesmo desempenho na publicação de periódicos de circulação internacional, muito provavelmente devido a dois fatores, a barreira do idioma e a quantidade de periódicos disponíveis para a publicação da área. Tabela 4.

Quando mudamos o período do recorte temporal 2000-2003 encontramos, um cenário bastante parecido com o anterior, entretanto, as humanas passam à frente da Saúde na publicação de livros com a publicação de 18.320 contra 17.537 da saúde, e mais uma vez as Exatas e da Terra se destacam no cenário internacional com 39.587 publicações contra 23.899 da Saúde e uma reduzida publicação das Humanas contabilizando apenas 4.407. Notase que o mesmo cenário ocorre no período de 2003-2006. Tabelas 5 e 6. 
Tabela 4. Número de autores e da produção científica dos pesquisadores doutores segundo grande área - Censos 1998-2001

\begin{tabular}{|c|c|c|c|c|c|c|}
\hline \multirow{3}{*}{ Grande área } & \multicolumn{6}{|l|}{ 1998-2001 } \\
\hline & \multirow{2}{*}{$\begin{array}{c}\text { Total } \\
\text { de } \\
\text { autores }\end{array}$} & \multicolumn{2}{|c|}{$\begin{array}{l}\text { Artigos completos publicados } \\
\text { em periódicos especializados }\end{array}$} & \multirow{2}{*}{$\begin{array}{c}\text { Trabalhos } \\
\text { completos } \\
\text { publicados em } \\
\text { anais de eventos }\end{array}$} & \multicolumn{2}{|c|}{$\begin{array}{l}\text { Livros e } \\
\text { capítulos } \\
\text { de livros } \\
\text { publicados }\end{array}$} \\
\hline & & $\begin{array}{l}\text { Circulação } \\
\text { nacional } \\
(1)\end{array}$ & $\begin{array}{l}\text { Circulação } \\
\text { internac. } \\
\text { (2) }\end{array}$ & & Livros & $\begin{array}{l}\text { Capítulos } \\
\text { de livros }\end{array}$ \\
\hline Ciências Agrárias & 4.999 & 27.488 & 9.965 & 28.399 & 1.495 & 5.803 \\
\hline Ciências Biológicas & 5.791 & 12.694 & 27.574 & 10.754 & 737 & 5.657 \\
\hline Ciências da Saúde & 5.698 & 26.102 & 17.688 & 13.189 & 1.651 & 10.831 \\
\hline Ciências Exatas e da Terra & 5.991 & 8.305 & 34.231 & 22.072 & 738 & 2.849 \\
\hline Ciências Humanas & 5.243 & 16.763 & 2.968 & 13.632 & 3.329 & 10.537 \\
\hline Ciências Sociais Aplicadas & 2.642 & 8.178 & 1.332 & 11.867 & 1.586 & 4.161 \\
\hline Engenharias & 5.851 & 7.430 & 14.789 & 60.319 & 870 & 3.495 \\
\hline Linguística, Letras e Artes & 1.534 & 5.132 & 814 & 3.716 & 975 & 3.227 \\
\hline Todas as grandes áreas & 32.839 & 96.021 & 91.263 & 139.761 & 10.015 & 40.152 \\
\hline
\end{tabular}

Fonte: Censos $\mathrm{CNPq}$

Tabela 5: Número de autores e da produção científica dos pesquisadores doutores segundo grande área - Censos 2000-2003

\begin{tabular}{|c|c|c|c|c|c|c|}
\hline \multirow{3}{*}{ Grande área } & \multicolumn{6}{|c|}{$2000-2003$} \\
\hline & \multirow{2}{*}{$\begin{array}{l}\text { Total } \\
\text { de } \\
\text { autores }\end{array}$} & \multicolumn{2}{|c|}{$\begin{array}{l}\text { Artigos completos publicados } \\
\text { em periódicos especializados }\end{array}$} & \multirow{2}{*}{$\begin{array}{l}\text { Trabalhos } \\
\text { completos } \\
\text { publicados em } \\
\text { anais de eventos }\end{array}$} & \multicolumn{2}{|c|}{$\begin{array}{l}\text { Livros e } \\
\text { capítulos } \\
\text { de livros } \\
\text { publicados }\end{array}$} \\
\hline & & $\begin{array}{l}\text { Circulação } \\
\text { nacional } \\
\text { (1) }\end{array}$ & $\begin{array}{l}\text { Circulação } \\
\text { internac. } \\
\text { (2) }\end{array}$ & & Livros & $\begin{array}{l}\text { Capítulos } \\
\text { de livros }\end{array}$ \\
\hline Ciências Agrárias & 6.795 & 44.277 & 12.099 & 42.408 & 1.944 & 8.077 \\
\hline Ciências Biológicas & 7.919 & 27.680 & 31.413 & 17.049 & 1.209 & 8.647 \\
\hline Ciências da Saúde & 8.700 & 46.725 & 23.899 & 19.179 & 2.330 & 17.537 \\
\hline Ciências Exatas e da Terra & 7.883 & 17.609 & 39.587 & 30.137 & 1.074 & 4.590 \\
\hline Ciências Humanas & 7.874 & 25.989 & 4.407 & 25.133 & 4.840 & 18.320 \\
\hline Ciências Sociais Aplicadas & 4.625 & 15.822 & 2.440 & 24.121 & 2.880 & 8.969 \\
\hline Engenharias & 8.072 & 14.856 & 18.593 & 88.507 & 1.244 & 5.385 \\
\hline Linguística, Letras e Artes & 2.479 & 8.519 & 1.237 & 6.220 & 1.635 & 5.639 \\
\hline Todas as grandes áreas & 46.117 & 165.571 & 105.898 & 208.392 & 14.618 & 64.629 \\
\hline
\end{tabular}

Fonte: Censos CNPq

Rev. Eletr de Cien. Tecnol e Inova, Rio de Janeiro, Vol. 10: 1-24, abril/2020. 


\begin{tabular}{|c|c|c|c|c|c|c|}
\hline \multirow{3}{*}{ Grande área } & \multicolumn{6}{|c|}{$2003-2006$} \\
\hline & \multirow{2}{*}{$\begin{array}{c}\text { Total } \\
\text { de } \\
\text { autores }\end{array}$} & \multicolumn{2}{|c|}{$\begin{array}{l}\text { Artigos completos publicados } \\
\text { em periódicos especializados }\end{array}$} & \multirow{2}{*}{$\begin{array}{c}\text { Trabalhos } \\
\text { completos } \\
\text { publicados em } \\
\text { anais de eventos }\end{array}$} & \multicolumn{2}{|c|}{$\begin{array}{c}\text { Livros e } \\
\text { capítulos } \\
\text { de livros } \\
\text { publicados }\end{array}$} \\
\hline & & $\begin{array}{c}\text { Circulação } \\
\text { nacional } \\
(1)\end{array}$ & $\begin{array}{l}\text { Circulação } \\
\text { internac. } \\
\text { (2) }\end{array}$ & & Livros & $\begin{array}{l}\text { Capítulos } \\
\text { de livros }\end{array}$ \\
\hline Ciências Agrárias & 7.876 & 50.921 & 21.257 & 41.870 & 2.212 & 10.448 \\
\hline Ciências Biológicas & 9.213 & 26.625 & 53.482 & 19.147 & 1.487 & 12.317 \\
\hline Ciências da Saúde & 10.897 & 57.105 & 47.640 & 23).026 & 2.897 & 26.425 \\
\hline Ciências Exatas e da Terra & 8.548 & 16.658 & 52.153 & 35.856 & 1.364 & 5.766 \\
\hline Ciências Humanas & 10.246 & 35.837 & 5.698 & 47.906 & 6.308 & 28.633 \\
\hline Ciências Sociais Aplicadas & 6.095 & 23.541 & 3.565 & 42.666 & 3.828 & 14.227 \\
\hline Engenharias & 9.038 & 16.748 & 27.175 & 111.736 & 1.562 & 6.601 \\
\hline Linguística, Letras e Artes & 3.292 & 11.045 & 1.472 & 10.500 & 2.120 & 9.105 \\
\hline Todas as grandes áreas & 55.214 & 196.345 & 165.056 & 274.851 & 18.338 & 94.017 \\
\hline \multicolumn{7}{|l|}{ Fonte: Censos CNPq } \\
\hline \multirow{3}{*}{ Grande área } & \multicolumn{6}{|c|}{$2007-2010$} \\
\hline & \multirow{2}{*}{$\begin{array}{c}\text { Total } \\
\text { de } \\
\text { autores }\end{array}$} & \multicolumn{2}{|c|}{$\begin{array}{l}\text { Artigos completos publicados } \\
\text { em periódicos especializados }\end{array}$} & \multirow{2}{*}{$\begin{array}{c}\text { Trabalhos } \\
\text { completos } \\
\text { publicados em } \\
\text { anais de eventos }\end{array}$} & \multicolumn{2}{|c|}{$\begin{array}{c}\text { Livros e } \\
\text { capítulos } \\
\text { de livros } \\
\text { publicados }\end{array}$} \\
\hline & & $\begin{array}{c}\text { Circulação } \\
\text { nacional } \\
\text { (1) }\end{array}$ & $\begin{array}{l}\text { Circulação } \\
\text { internac. } \\
\text { (2) }\end{array}$ & & Livros & $\begin{array}{l}\text { Capítulos } \\
\text { de livros }\end{array}$ \\
\hline Ciências Agrárias & 10.889 & 69.166 & 39.073 & 42.538 & 2.329 & 15.178 \\
\hline Ciências Biológicas & 12.376 & 38.552 & 84.397 & 18.360 & 1.940 & 16.960 \\
\hline Ciências da Saúde & 14.571 & 78.027 & 88.441 & 22.412 & 3.219 & 29.639 \\
\hline Ciências Exatas e da Terra & 10.707 & 24.503 & 64.777 & 39.188 & 1.690 & 8.236 \\
\hline Ciências Humanas & 15.220 & 56.469 & 9.582 & 81.047 & 9.126 & 49.970 \\
\hline Ciências Sociais Aplicadas & 8.545 & 36.639 & 5.724 & 60.804 & 5.466 & 25.469 \\
\hline Engenharias & 11.673 & 25.569 & 39.559 & 131.453 & 2.092 & 10.875 \\
\hline Linguística, Letras e Artes & 4.780 & 15.553 & 1.649 & 17.048 & 3.110 & 14.966 \\
\hline Todas as grandes áreas & 88.761 & 344.478 & 333.202 & 412.850 & 28.972 & 171.293 \\
\hline
\end{tabular}

Fonte: Censos CNPq

Notas: Não há dupla contagem nos quantitativos da produção, excetuando-se os trabalhos de co-autorias entre pesquisadores participantes do Diretório; Grande área do grupo de que o pesquisador participa.

Rev. Eletr de Cien. Tecnol e Inova, Rio de Janeiro, Vol. 10: 1-24, abril/2020. 
A Tabela 7 mostra que a Saúde volta a ocupar o primeiro lugar no ranking nacional quando comparada às demais áreas de conhecimento com um crescimento exponencial de artigos publicados em periódicos de veiculação internacional de 17.688 para 88.441 artigos.

O Diretório dos Grupos de Pesquisa no Brasil constitui-se no inventário dos grupos de pesquisa em atividade no país. Suas bases de dados contêm informações sobre os recursos humanos constituintes dos grupos, as linhas de pesquisa em andamento, as especialidades do conhecimento, os setores de atividade envolvidos, a produção científica, tecnológica e artística dos pesquisadores e estudantes que integram os grupos e aos padrões de interação com o setor produtivo. Esses grupos estão localizados em universidades, instituições isoladas de ensino superior, institutos de pesquisa científica, institutos tecnológicos, laboratórios de pesquisa e desenvolvimento de empresas estatais ou ex-estatais, e em algumas organizações não-governamentais com atuação em pesquisa. ${ }^{7-9}$

O Diretório foi iniciado no CNPq em 1992 e, desde então, numa frequência quase sempre bienal, a Agência torna disponível ao público em geral um censo da capacidade instalada de pesquisa no país, medida pelos grupos ativos em cada período. Dessa forma, cada grupo é situado no espaço e no tempo. A partir de 2002, o Diretório tornou o sistema disponível para atualização contínua da base de dados, denominada Base Corrente, porém mantendo a frequência bienal para a realização dos censos, que passaram a ser "fotografias" dessa base corrente. ${ }^{7}$

Os censos apresentam informações quantitativas sobre os grupos em suas diversas dimensões e oferecem recursos de buscas textuais sobre as bases de dados. Neste site, estão disponíveis os resultados dos Censos realizados a partir de 2000. Quando analisamos o número de instituições e de grupos de pesquisa no Brasil, segundo as regiões, encontramos os seguintes cenários: a região sudeste se destaca em todos os períodos avaliados, seguido pelas regiões sul e nordeste, consecutivamente. Este dado pode ser explicado pela maior quantidade de programas de pós-graduação na região sudeste em relação às demais regiões.

Rev. Eletr de Cien. Tecnol e Inova, Rio de Janeiro, Vol. 10: 1-24, abril/2020. 
Não obstante, o quadro 1 mostra a distribuição dos grupos de pesquisa segundo a grande área de conhecimento no senso de 2010 e o que chama atenção é a maior quantidade de grupos de pesquisa nas humanas, enquanto que a Saúde vem em segundo lugar. ${ }^{8}$

No entanto, quando analisamos o quadro 2 é que das 74 áreas de conhecimento predominantes nas atividades do ano de 2010, a enfermagem ocupa o $20^{\circ}$ lugar, sendo a $4^{\mathrm{a}}$ colocada na área de saúde com 482 grupos de pesquisa, o que equivale a 1,8\% do total de grupos de pesquisa das grandes áreas de conhecimento registradas no CNPq, estando atrás, apenas da Medicina, Saúde Coletiva e Odontologia, sucessivamente, $1^{\mathrm{a}}, 2^{\mathrm{a}}$ e $3^{\mathrm{a}}$ colocadas. Isso demonstra a força e o potencial da enfermagem diante do cenário nacional da pesquisa e da produção científica..$^{7-9,14}$

\begin{tabular}{|c|c|c|c|c|c|c|c|c|c|c|c|c|}
\hline \multirow{2}{*}{ Região } & \multicolumn{6}{|c|}{ Instituições } & \multicolumn{6}{|c|}{ Grupos (G) } \\
\hline & 2000 & 2002 & 2004 & 2006 & 2008 & 2010 & 2000 & 2002 & 2004 & 2006 & 2008 & 2010 \\
\hline Centro-Oeste & 12 & 18 & 25 & 32 & 33 & 38 & 636 & 809 & 1.139 & 1.275 & 1.455 & 1.965 \\
\hline Nordeste & 39 & 48 & 58 & 63 & 67 & 73 & 1.720 & 2.274 & 2.760 & 3.269 & 3.863 & 5.044 \\
\hline Norte & 13 & 19 & 31 & 37 & 41 & 41 & 354 & 590 & 770 & 933 & 1.070 & 1.433 \\
\hline Sudeste & 125 & 140 & 173 & 210 & 216 & 236 & 6.733 & 7.855 & 10.221 & 10.592 & 11.120 & 12.877 \\
\hline Sul & 49 & 58 & 70 & 84 & 84 & 92 & 2.317 & 3.630 & 4.580 & 4.955 & 5.289 & 6.204 \\
\hline Brasil & 224 & 268 & 335 & 403 & 422 & 452 & 11.760 & 15.158 & 19.470 & 21.024 & 22.797 & 27.523 \\
\hline
\end{tabular}

Quadro 1: Distribuição dos grupos de pesquisa segundo a grande área do conhecimento predominante nas atividades do grupo, 2010.

\begin{tabular}{|c|c|c|c|c|}
\hline \multicolumn{2}{|c|}{ Grande área do conhecimento } & Grupos & $\%$ & \multirow[t]{2}{*}{$\%$ acumulado } \\
\hline Humanidades & Ciências Humanas & 5.387 & 19,6 & \\
\hline Ciências da Vida & Ciências da Saúde & 4.573 & 16,6 & 36,2 \\
\hline Ciências da Natureza & Engenharias & 3.548 & 12,9 & 49,1 \\
\hline Humanidades & Ciências Sociais Aplicadas & 3.438 & 12,5 & 61,6 \\
\hline Ciências da Vida & Ciências Biológicas & 3.108 & 11,3 & 72,9 \\
\hline Ciências da Natureza & Ciências Exatas e da Terra & 2.934 & 10,7 & 83,5 \\
\hline Ciências da Vida & Ciências Agrárias & 2.699 & 9,8 & 93,3 \\
\hline Humanidades & Lingüística, Letras e Artes & 1.836 & 6,7 & 100,0 \\
\hline Total & & 27.523 & 100,0 & - \\
\hline
\end{tabular}

Fonte: Censos CNPq

Rev. Eletr de Cien. Tecnol e Inova, Rio de Janeiro, Vol. 10: 1-24, abril/2020. 
Quadro 2: Distribuição dos grupos de pesquisa segundo a área do conhecimento predominante nas atividades do grupo, 2010.

\begin{tabular}{|c|c|c|c|}
\hline Área do conhecimento & Grupos & $\%$ & $\%$ acumulado \\
\hline 1- Educação & 2.236 & 8,1 & 8,1 \\
\hline 2- Medicina & 1.437 & 5,2 & 13,3 \\
\hline Agronomia & 1.040 & 3,8 & 17,1 \\
\hline 3-Química & 1.036 & 3,8 & 20,9 \\
\hline 4- Direito & 776 & 2,8 & 23,7 \\
\hline 5- Ciência da Computação & 776 & 2,8 & 26,5 \\
\hline 6- Administração & 757 & 2,8 & 29,3 \\
\hline 7- Saúde Coletiva & 732 & 2,7 & 31,9 \\
\hline 8- Física & 731 & 2,7 & 34,6 \\
\hline 9- História & 690 & 2,5 & 37,1 \\
\hline 10- Letras & 672 & 2,4 & 39,5 \\
\hline 11- Psicologia & 669 & 2,4 & 42,0 \\
\hline 12- Lingüística & 652 & 2,4 & 44,3 \\
\hline 13- Odontologia & 554 & 2,0 & 46,4 \\
\hline 14- Engenharia Elétrica & 547 & 2,0 & 48,3 \\
\hline 15- Geociências & 538 & 2,0 & 50,3 \\
\hline 16-Artes & 512 & 1,9 & 52,2 \\
\hline 17- Medicina Veterinária & 498 & 1,8 & 54,0 \\
\hline 18- Educação Física & 494 & 1,8 & 55,8 \\
\hline 19- Ecologia & 490 & 1,8 & 57,5 \\
\hline 20- Enfermagem & 482 & 1,8 & 59,3 \\
\hline
\end{tabular}

Fonte: Censos CNPq

Quando passamos a analisar o comportamento da enfermagem nos cenários nacional e mundial, juntamente com outras áreas do conhecimento, podemos identificar que, de acordo com a Scimago no período de 2011 a 2012, essa área de conhecimento, muito embora permaneça diretamente ligada a medicina, ainda assim, já se configura como área de conhecimento científico já consolidada, conforme podemos verificar no pictograma 1 , onde o tamanho da esfera/nó é diretamente proporcional ao volume de publicação da área. A mesma relação ocorre com a linha de ligação entre as áreas, quanto mais densa for, maior é a publicação em co-autoria. ${ }^{10-14}$ Nesse sentido, é possível identificar que diferentemente 
da imunologia e da bioquímica, a enfermagem possui uma linha menos densa o que significa dizer que as duas outras áreas de conhecimento estão muito mais colaborativas com a medicina que a enfermagem. Outra análise possível, a partir do referido pictograma, é que a enfermagem ainda não tem outras áreas de conhecimento a ela interligadas, tal como podemos evidenciar com a medicina, a genética e a biologia molecular que se encontram ramificadas em outras áreas de conhecimento. ${ }^{16-20}$

Pictograma 1 conformação da colaboração entre a enfermagem e as demais áreas de conhecimento

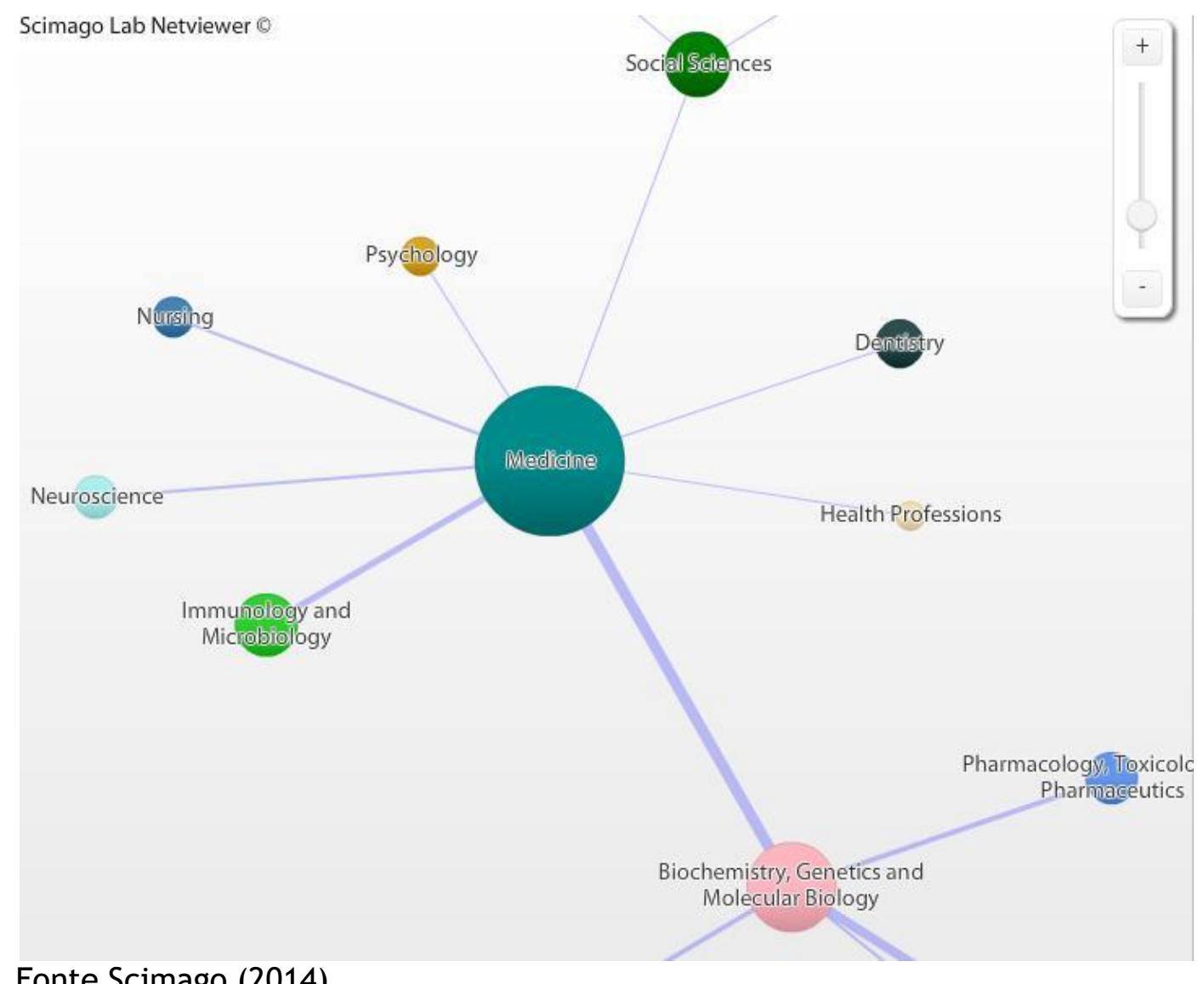

Fonte Scimago (2014)

Quando comparamos a enfermagem brasileira com outros países da América Latina, Argentina, Chile e Colômbia, fica evidente o domínio do Brasil na quantidade de documentos publicados desde 1996 até 2012, conforme o gráfico 1. O mesmo ocorre com o fator de impacto (índice $\mathrm{H}$ ), entretanto, muito prejudicado na colaboração internacional, gráficos 2 e 3 respectivamente. Isso reforça a necessidade de a enfermagem brasileira buscar cada vez 
mais a colaboração de pesquisadores internacionais, visto que, o quadro atual não é favorável à internacionalização das nossas produções.

\section{CONCLUSÃO}

O estudo permitiu um panorama da produção científica da enfermagem brasileira, na perspectiva da enfermagem do trabalho e o referente "qualidade total" como possível de ser investigado no plano da enfermagem, indexados aos bancos de dados Scopus e Scimago, duas gigantescas e respeitadas bases de dados.

A análise bibliométrica permite afirmar com tranquilidade que existe um crescimento notável no número de instituições e de grupos de pesquisa na grande área da saúde, entretanto, ainda incapaz de ultrapassar as ciências humanas no recorte temporal estudado (200-2010). O mesmo cenário foi possível quando da análise do quantitativo de pesquisadores doutores e estudantes, observa-se que o crescimento foi proporcional.

Considerando que o "termômetro" mais utilizado para mensuração da produção científica é a publicação de artigos, foi possível evidenciar que no âmbito nacional a área da saúde lidera, entretanto, quando o cenário é a circulação internacional, as ciências exatas e da terra apresentam o dobro de artigos publicados pela área da saúde e quase dez (10) vezes mais que as ciências humanas.

Das vinte (20) áreas de conhecimento, a enfermagem está entre as cinco com o maior quantitativo de grupos de pesquisa da grande área saúde. Na América latina há predominância da enfermagem brasileira no quesito quantidade de documentos até 2012, inclusive com maior índice H. Mas, quando se compara a cooperação internacional entre o Brasil, Argentina, Chile e Colômbia, o Brasil mostra um decréscimo em seu desempenho, cujo pico ocorreu em 1997. Existe uma grande possibilidade de mudança deste cenário com o Programa do Governo Federal Ciência Sem Fronteiras, mesmo já tendo iniciado em 2011.

$\mathrm{Na}$ grande área de saúde a produção científica de enfermagem com o descritor “enfermagem do trabalho” pode ser considerada pequena quando comparada à recuperação 
de documentos com o descritor "enfermagem”. No cenário mundial o Brasil ocupa o $8^{\circ}$ lugar, perdendo três (3) posições quando comparado com o descritor “enfermagem”. Merece destaque a posição da Universidade de São Paulo que perde, apenas, para VA Medical Center dos Estados Unidos da América, inclusive, com o descritor “enfermagem do trabalho".

O Brasil mostra um baixo desempenho quando a busca ocorre com o descritor "enfermagem do trabalho e qualidade total". Com este descritor a enfermagem brasileira deixa o top ten. Isso mostra que a referente qualidade total na perspectiva da enfermagem do trabalho ainda precisa ser melhor e mais abordada/pesquisada nas instituições de ensino e pesquisa, principalmente, nos cursos de pós-graduação - mestrado e doutorado.

\section{REFERÊNCIAS}

1. ABRAMO, G.; D `ANGELO, C.A.;DI COSTA, F. Research collaboration and productivity: is there correlation? High Education, v. 57, p. 155-171, 2009.

2. Bachelard, G. A formação do espírito científico: contribuição para uma psicanálise do conhecimento. Rio de Janeiro: Contraponto, 1996. 314 p.

3. Ferreira, M.C.G. Avaliação de periódicos científicos. Disponível em: http://www.biblioteca.ufc.br/cecilia.ppt.

4. Da Silva, V R F, Silva, CRL, Cheng, C et al. Análise bibliométrica de artigos sobre cateterização venosa periférica em pediatria. Cogitare Enfermagem, [S.l.], v. 24, abr. 2019. ISSN 2176-9133. Disponível em: <https://revistas.ufpr.br/cogitare/article/view/59232>. Acesso em: 05 abr. 2020. doi: http://dx.doi.org/10.5380/ce.v24i0.59232.

5. Fernandes, MRCC, Silva, CRL. Produtividade científica acerca da neoplasia maligna bucal e da reabilitação bucal: uma análise bibliométrica. Cogitare Enfermagem, [S.l.], v. 22, n. 4, out. 2017. ISSN 2176-9133. Disponível em: <https://revistas.ufpr.br/cogitare/article/view/50046>. Acesso em: 05 abr. 2020. doi: http://dx.doi.org/10.5380/ce.v22i4.50046. 
6. Fonseca, EN. Bibliometria: teoria e pratica / Edson da Fonseca, organizador; textos de Paul Otlet ... [et al.]; tradução e Alda Baltar, Ivanilda Fernandes Costa Rolim, Jose Paulo Paes. São Paulo : Cultrix, 1986.

7. Gil, AC. Métodos e Técnicas de Pesquisa Social 5. ed. São Paulo:Atlas, 2006.

8. Grácio, M.C.C.; Oliveira, E.F.T. Produção e Comunicação da Informação em CT\&l GT7 da ANCIB: análise bibliométrica no período 2003/2009, 2011.

9. Guedes, V; Borschiver, S. Bibliometria: uma ferramenta estatística para a gestão da informação e do conhecimento, em sistemas de informação, de comunicação e de avaliação cientifica e tecnológica. In: CINFORM - Encontro Nacional de Ciencia Da Informação, 6., 2005, Salvador. Anais. Salvador: ICI/UFBA, 2005.

10. Hoekman, J.; Frenken, K.; Tijssen, R. J. W. Research collaboration at a distance: changing spatial patterns of scientific collaboration within Europe. Research Policy, v.30, p.662-673, 2010. https://doi.org/10.1016/j.respol.2010.01.012

11. Leopardi, M.T. Metodologia da pesquisa na saúde. Santa Maria: Pallotti, 2001.

12. Narin, Francis. Evaluative bibliometrics: the use of publication and citation analysis in the evaluation of scientific activity. Cherry Hill, N. J.: Computer Horizons, 1976.

13. Organização para a Cooperação Econômica (OECE, 2004)

14. Pinheiro, LVR. Lei de Bradford: uma reformulação conceitual. Ciência da Informação, Brasília, v. 12, n. 2, p. 59-80, jul./dez. 1983.

15. RAO, I.K. Métodos quantitativos em biblioteconomia e ciência da informação. Brasília: Associação dos Bibliotecários do Distrito Federal, 1986.

16. Vanti, N.A.P. Da bibliometria à webometria: uma exploração conceitual dos mecanismos utilizados para medir o registro da informação e a difusão do conhecimento. Ciência da Informação, Brasília, v.31, n.2, maio/ago.2002. https://doi.org/10.1590/S010019652002000200016 
17. Vaz, D.C., SILVA, C. R. L., Figueiredo, N, M. A. A Utilização da Bibliometria na Análise do Referente Conforto.

18. Voos, H. Lotka and Information Science. Journal of the American Society for Information Science, 1974. https://doi.org/10.1002/asi.4630250410

19. Wagner, C. S.; Leydesdorff, L. Network structure, self-organization, and the growth of international collaboration in science. Reserch Policy, v.34, n.10, p. 1608-1618, 2005. https://doi.org/10.1016/j.respol.2005.08.002

20. http://www.scimagojr.com/countryrank.php.

Rev. Eletr de Cien. Tecnol e Inova, Rio de Janeiro, Vol. 10: 1-24, abril/2020. 